\title{
Targeting Translation Control with p70 S6 Kinase I Inhibitors to Reverse Phenotypes in Fragile X Syndrome Mice
}

\author{
Aditi Bhattacharya ${ }^{1,6}$, Maggie Mamcarz', Caitlin Mullins',2, Ayesha Choudhury',3, Robert G Boyle ${ }^{4}$, \\ Daniel G Smith ${ }^{5}$, David W Walker ${ }^{4}$ and Eric Klann*,I \\ 'Center for Neural Science, New York University, New York, NY, USA; ${ }^{2}$ New York University Neuroscience Institute, New York University Langone \\ Medical Center, New York, NY, USA; ${ }^{3}$ Department of Psychology, Hunter College, City University of New York, New York, NY, USA; ${ }^{4}$ Sentinel \\ Oncology, Cambridge, UK; ${ }^{5}$ Autism Speaks, Boston, MA, USA
}

\begin{abstract}
Aberrant neuronal translation is implicated in the etiology of numerous brain disorders. Although mTORCI-p70 ribosomal S6 kinase I (S6KI) signaling is critical for translational control, pharmacological manipulation in vivo has targeted exclusively $\mathrm{mTORCI}$ due to the paucity of specific inhibitors to S6KI. However, small molecule inhibitors of S6KI could potentially ameliorate pathological phenotypes of diseases, which are based on aberrant translation and protein expression. One such condition is fragile $X$ syndrome (FXS), which is considered to be caused by exaggerated neuronal translation and is the most frequent heritable cause of autism spectrum disorder (ASD). To date, potential therapeutic interventions in FXS have focused largely on targets upstream of translational control to normalize FXSrelated phenotypes. Here we test the ability of two S6KI inhibitors, PF-470867I and FS- I I5, to normalize translational homeostasis and other phenotypes exhibited by FXS model mice. We found that although the pharmacokinetic profiles of the two S6KI inhibitors differed, they overlapped in reversing multiple disease-associated phenotypes in FXS model mice including exaggerated protein synthesis, inappropriate social behavior, behavioral inflexibility, altered dendritic spine morphology, and macroorchidism. In contrast, the two inhibitors differed in their ability to rescue stereotypic marble-burying behavior and weight gain. These findings provide an initial pharmacological characterization of the impact of S6KI inhibitors in vivo for FXS, and have therapeutic implications for other neuropsychiatric conditions involving aberrant mTORCI-S6KI signaling.

Neuropsychopharmacology (2016) 4I, I99I-2000; doi: I 0. I 038/npp.2015.369; published online 20 January 2016
\end{abstract}

\section{INTRODUCTION}

Precise temporal and spatial control of translation is critical for proper neural function and behavior. Major brain disorders such as autism, schizophrenia, and Alzheimer's disease have been shown to involve aberrant neuronal translation (Hoeffer and Klann, 2010; Lipton and Sahin, 2014). The mammalian target of rapamycin complex 1 (mTORC1) along with its downstream effectors, eIF4Ebinding protein (4E-BP) and p70 ribosomal S6 kinase 1 (S6K1), is a critical signaling nexus that controls general and transcript-specific translation (Costa-Mattioli et al, 2009). S6K1 regulates both translation initiation and elongation: by stimulating eIF4A helicase activity via the phosphorylation of eIF4B, it promotes cap-dependent initiation, and via the phosphorylation and inhibition of eEF2 kinase, S6K1 enhances the activity of eEF2 to promote

\footnotetext{
* Correspondence: Dr E Klann, Center for Neural Science, New York University, 4 Washington Place, Room 809, New York, NY I0003, USA, Tel: + I 212992 9769, E-mail: eklann@cns.nyu.edu

${ }^{6}$ Current address: Center for Brain Development and Repair, Institute for Stem Cell Biology and Regenerative Medicine, National Center for Biological Sciences, GKVK Campus, Bangalore 560065, India

Received 12 October 20 15; revised II December 2015; accepted 22 December 2015; accepted article preview online 28 December 2015
}

translocation of the nascent polypeptide chain on the ribosome (Magnuson et al, 2012).

In order to reduce exaggerated protein synthesis, the majority of pharmacological and clinical interventions in both patients and animal models of disease have focused on disruption of mTORC1 with rapamycin or related analogs (Eyre et al, 2014; Lipton and Sahin, 2014). S6K1 and phosphorylation of its substrate ribosomal protein S6 traditionally have been studied as a read out of mTORC1 activity. Recently, S6K1 has become a therapeutic target for certain cancers (Holz, 2012; Tolcher et al, 2014), but its importance in etiology of brain disorders remains poorly understood. Before the introduction of PF-4708671 (Pearce et al, 2010), selective S6K1 inhibitors were unavailable for basic research use. In addition, neuronal translational control is likely different from that in cancer cells and thus, there is a need for the characterization of S6K1 inhibitors in neurobiology.

Fragile X syndrome (FXS) is perhaps the best known brain disorder associated with aberrant translation as a core molecular phenotype. Because FXS is the single largest cause of genetically inherited autism spectrum disorder (ASD) and intellectual disability (ID), FXS has been studied intensely. Caused by a CGG repeat expansion in the $5^{\prime}$ UTR of the FMR1 gene (Santoro et al, 2012), FXS results in the loss of 
the translational regulator fragile $\mathrm{X}$ mental retardation protein (FMRP; Darnell and Klann, 2013). The lack of FMRP also is associated with enhanced activation of the phosphoinositide 3-kinase (PI3K), mTORC1, and extracellular signalregulated kinase (ERK) signaling pathways (Gross et al, 2010, Osterweil et al, 2010; Sharma et al, 2010); all of which signal downstream to S6K1. Consistent with upregulation of PI3K, mTORC1, and ERK signaling, increased S6K1 signaling has been reported in hippocampal lysates from FXS mice, as well as in lymphocytes, postmortem brain, and primary fibroblasts from FXS patients (Hoeffer et al, 2012; Kumari et al, 2014). Given its ubiquitous role in translational control, we previously hypothesized that targeting S6K1 would correct pathophysiologies associated with FXS, and then demonstrated that genetically reducing S6K1 corrects a range of phenotypes displayed by Fmr1 knockout (Fmr1 KO) mice (Bhattacharya et al, 2012). Consequently, we sought to address the combined need of characterizing the effectiveness of S6K1 inhibitors in vivo in the CNS, and to extend the results of genetic deletion studies in a more therapeutically relevant direction by evaluating the ability of two novel and selective inhibitors of S6K1 to reverse phenotypes displayed by Fmr1 KO mice. Our results support the viability of S6K1 inhibitors as a therapeutic intervention for FXS and provide information on how S6K1 blockade affects translation in the brain in vivo.

\section{MATERIALS AND METHODS}

\section{Mice and Experimental Design}

Fmr1 KO mice were bred and maintained as described in Sharma et al (2010) and Bhattacharya et al (2012) by crossing female $\mathrm{X}^{\mathrm{Fmr1+}} \mathrm{X}^{\mathrm{Fmr1}-}$ with $\mathrm{X}^{\mathrm{Fmr1+}} / \mathrm{Y}$ male mice on a C57/Bl6 background (originally from Jackson Labs, ME, USA). The colony was routinely backcrossed to control for inbreedingrelated issues. All procedures were in accordance with protocols approved by the New York University Animal Welfare Committee and followed the NIH Guidelines for the care and use of animals in research.

At the commencement of this study, published data on PF-4708671 only extended to cell lines, whereas maximal tolerated dose (MTD) assays were being carried out in-house for separate cancer models for FS-115 by Sentinel Oncology. Therefore, initial biochemical experiments and dose optimization were first carried out in wild-type (WT) C57/Bl6 mice with appropriate controls. When we had determined the ideal dose and time durations of treatments, the same experiments were carried out with Fmr1 KO (FXS model) mice. Statistical tests in Figure 1 reflect this differential distribution of treatment groups, while Figure 3 onwards reflect a set dose and time duration in which yolked sets of WT and Fmr1 KO were used. While optimizing dose and treatment regimes, mice were monitored daily for weight and general home-cage activity by two independent experimenters with veterinary consultation on call.

\section{Compound Pharmacodynamics and Pharmacokinetics}

PF-4708671 was acquired under a compound transfer agreement from Pfizer and made available via SigmaAldrich, MO, USA. FS-115 was obtained from Sentinel
Oncology, Cambridge, UK under a memorandum of understanding. For acute application on brain slices and cortical lysates, both reagents were dissolved in DMSO (Sigma-Aldrich, master stock $10 \mathrm{mM}$ ) and used at a concentration of $10 \mu \mathrm{M}$. For intraperitoneal (i.p.) injections of PF-4708671, a vehicle consisting of saline $+5 \% \mathrm{v} / \mathrm{v}$ of Tween-80 (Sigma-Aldrich) was used, which when combined with drug and sonicated vigorously yielded a fine suspension. We dissolved FS-115 in a vehicle comprising of equal volumes of $5 \% \mathrm{DMSO}+0.1 \mathrm{~N} \mathrm{HCl}$ (Fischer Scientific) $+20 \%$ 2 -(hydroxypropyl)- $\beta$-cyclodextrin (Sigma-Aldrich, $\mathrm{pH}$ stabilized to 6.5-7), which upon sonication with drug yielded a clear yellow solution.

The pharmacokinetic profile of FS-115 was determined with a discrete oral/i.v. PK experiment in male CD-1 mice. Following a single dose of FS-115 at $10 \mathrm{mg} / \mathrm{kg}$ p.o. and $2 \mathrm{mg} / \mathrm{kg}$ i.v., plasma was sampled at eight time points $(n=3$ mice per time point) over $24 \mathrm{~h}$, followed by protein precipitation using methanol and analysis by LC-MS/MS. Brain-penetrance of FS-115 was determined by a discrete oral PK experiment in male CD-1 mice. Following a single dose of FS-115 at $25 \mathrm{mg} / \mathrm{kg}$ p.o., plasma and brain was sampled at three time points $(0.5,1.1$, and $2.6 \mathrm{~h})$, followed by protein precipitation and analysis by LC-MS/MS.

The FS-115 kinase inhibitor profile was generated by screening against a selection of isolated human kinases offered by Merck Millipore as part of their KinaseProfiler Service Assay. The data for FS-115 described in Supplementary Table S1 represent the mean from two replicates. The concentration of ATP used was at $\mathrm{Km}$ of the respective kinase. Pharmacokinetic profile of PF-4708671 was generated in-house by Pfizer Neuroscience Research facility. Male C57B16 mice were used and three to four mice per time point were used to derive PK values. Kinase profile values were adapted from Pearce et al (2010).

\section{Western Blotting}

All western blotting procedures were carried out as described previously in Bhattacharya et al (2012). The following antibodies were used: p-S6 240/44 (Cell Signaling Technologies (CST), MA, USA), 1:2000; total S6 (CST), 1:1500; p-eEF2 (Thr 56, CST), 1:1000; total eEF2 (CST), 1:1000; and p-eIF4B (Abcam), $\beta$-tubulin and actin (CST) $1: 10000$. Anti-rabbit and anti-mouse-HRP-tagged antibodies (Promega, WI, USA) were diluted at $1: 10000$.

\section{Measurement of De Novo Protein Synthesis using FUNCAT}

FUNCAT (fluorescent noncanonical amino acid tagging), a non-radioactive amino acid labeling method to measure de novo translation was modified for use in intact brain slices as described previously (Bowling et al, 2016).

\section{BONCAT Assay in Cortical Lysates}

The snapshot BONCAT assay for measuring protein synthesis rates in cortical lysates was adapted from previous studies (Udagawa et al, 2013; Bowling et al, 2016). Briefly, Fmr1 KO mice and WT littermates between 3 and 4 months of age were killed, and the brains were collected 
a
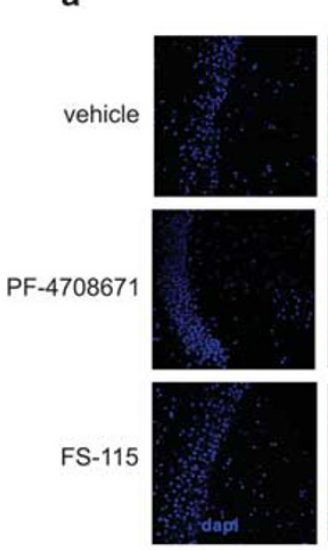

wild-type
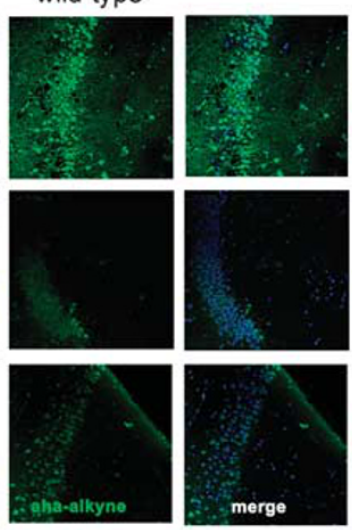

C

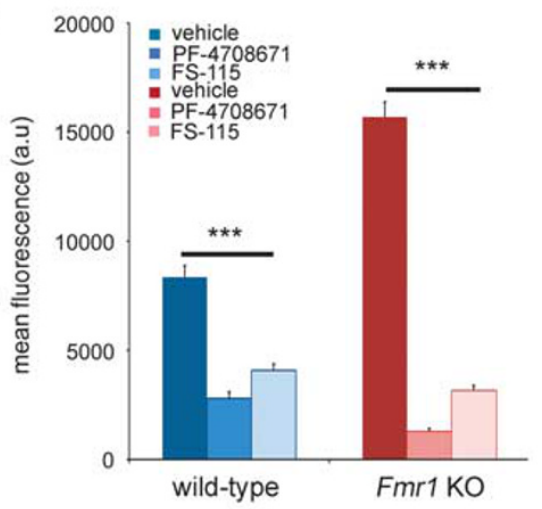

b
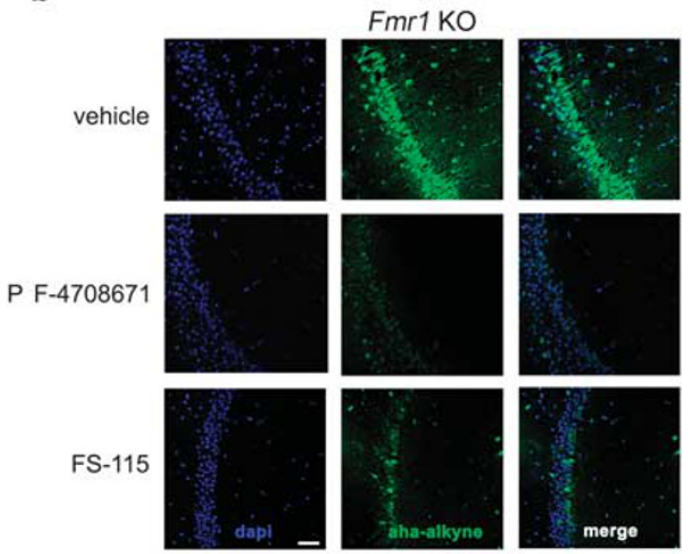

d

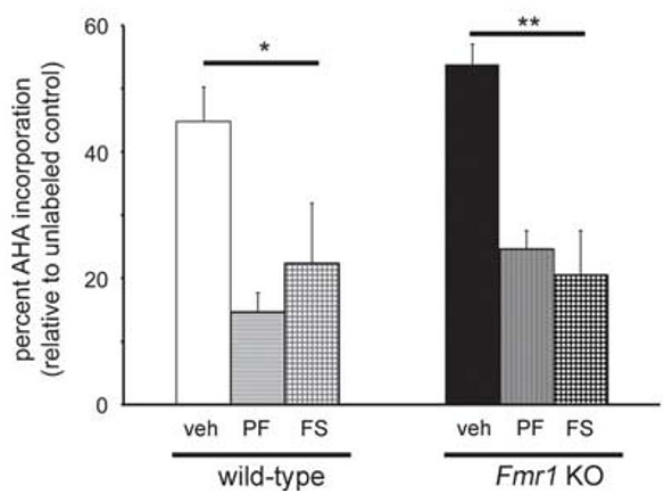

Figure I S6KI inhibition decreases protein synthesis and elongation in the brain. (a and b) De novo protein synthesis in hippocampal slices from wild-type (WT) and Fmrl knockout (Fmrl KO) mice in the presence of vehicle, PF-470867I and FS-I I 5 measured using FUNCAT. Scale bar, $50 \mu \mathrm{m}$. (c) Quantification of the effect of PF-470867I and FS-II5 on protein synthesis in hippocampal slices from WT and Fmr I KO mice using FUNCAT. $n=60$ neurons, sampled across three mice and three slices per treatment. Protein synthesis measurements from WT and Fmr I KO mice were conducted separately, but all treatments (vehicle, PF-470867I, and FS-I I 5) were done as yoked sets. ***** $<0.00$ I with a one-way ANOVA. (d) BONCAT AHA incorporation in cortical lysates from mice treated with vehicle, PF-470867I, or FS-I I5. $n=3-4$ mice per genotype per treatment. $* p<0.05$, $* *$ $p<0.0$ I by single factor ANOVA. Error bars represent SEM in (c) and (d). See also Supplementary Figure SIC-E.

and rinsed in ice-cold $1 \times$ HBSS (Invitrogen) $+10 \mathrm{mM}$ HEPES-KOH. Cerebral cortices were dissected rapidly and homogenized in lysis buffer $(25 \mathrm{mM}$ HEPES-KOH ( $\mathrm{pH}$ 7.4), $50 \mathrm{mM} \mathrm{KCl}, 1.5 \mathrm{mM} \mathrm{MgCl}_{2}, 0.5 \mathrm{mM}$ DTT, plus protease inhibitor cocktail (Sigma-Aldrich) and 40 units $/ \mathrm{ml}$ RNaseOUT (Invitrogen)). The homogenate was centrifuged at low speed (2000 r.p.m.) for $10 \mathrm{~min}$ at $4{ }^{\circ} \mathrm{C}$, and the equal volume aliquots of the resultant supernatant were added to microcentrifuge tubes to which was added a creatine-based ATP regenerating mix (in $\mathrm{mM}: 125$ HEPES-KOH (pH 7.4), 10 ATP (Sigma-Aldrich), 2 GTP (Sigma-Aldrich), 100 creatine phosphate (Sigma-Aldrich), 2 spermidine (Sigma-Aldrich), plus $1 \mathrm{mg} / \mathrm{ml}$ creatine phosphokinase (Calbiochem, EMD Millipore, MA, USA), $0.2 \mathrm{mM}$ amino acids (less cys/met mix, Promega, WI, USA) and $1 \mathrm{mM}$ final concentration of AHA. In parallel aliquot either $10 \mu \mathrm{M}$ PF-4708671 or $10 \mu \mathrm{M}$ FS115 or vehicle were added after $5 \mathrm{~min}$ of adding AHA. For chronic-treated samples, cortical lysates were incubated for $15 \mathrm{~min}$ with AHA and then flash frozen.

The lysate then was warmed to $30^{\circ} \mathrm{C}$, and $30 \mu \mathrm{l}$ samples were taken at $0 \mathrm{~min}$ as unlabeled control and $15 \mathrm{~min}$ afterwards. These samples were flash frozen and stored for subsequent processing for BONCAT as described in Bowling et al (2016). Quantification was done by measuring the integrated signal intensity of the whole lane, subtracting it from intensity of lane containing sample collected at $t=0 \mathrm{~min}$ and normalized to non-specific biotin band at $110 \mathrm{kDa}$.

\section{Behavioral Test Battery}

Three- to four-month-old male mice in two independent cohorts were studied. The behavioral tests were conducted in increasing order of difficulty and stress ranging from marble-burying, social interaction, and Y-maze choice arm reversal. Because gavage can be stressful, we acclimatized all mice to saline-gavage 3 days before delivering either vehicle or FS-115. Mice were either injected or gavaged at the same time every day between 0900 and 1000 hours. During days of behavioral testing, mice were injected/gavaged and then taken to experimental rooms for habituation. All tests began within $90 \mathrm{~min}$ of either drug or vehicle being administered and were performed as described previously 
(Bhattacharya et al, 2012). Mice were coded and the experimenter was blind to genotype and treatment. Analyses and scoring were done by a separate individual who also was blind to genotype and treatment. For a more detailed description of the behavioral tests, please refer to the Supplementary Information.

\section{Neuroanatomy}

Twelve-week-old mice were used and neurons stained using Rapid Golgi kit (FD NeuroTechnologies, MD, USA) as described previously (Bhattacharya et al, 2012).

\section{Mean Body Weight and Testicular Weight Analyses}

All mice undergoing chronic treatment with either PF-4708671 or FS-115 for biochemical, anatomical, or behavioral experiments were weighed every day at a fixed time and analyses of this pooled set was used. Testes from mice being used for biochemical and anatomical experiment cohorts were used. Briefly, after the brain was removed and stored for further experiments, the testes were dissected out and weighed immediately to three decimal place accuracy.

\section{Analysis Software}

All calculations and measurements were compiled in MS Office Excel. Statistical tests were done using InStat software (GraphPad Software, CA, USA).

\section{RESULTS}

\section{S6K1 Inhibition Reduces Basal Translation in the Brain}

A key feature of an effective S6K1 inhibitor would be its ability to impact basal protein synthesis. Recently, we showed that FXS patient-specific fibroblasts treated acutely with an S6K1 inhibitor reduced protein synthesis (Kumari et al, 2014). To test the efficacy of S6K1 inhibitors in brain tissue, we first determined the optimal concentrations of PF-4708671 and FS-115 in WT hippocampal slices by measuring the phosphorylation of the S6K1 substrate ribosomal protein S6 (Supplementary Information; Supplementary Figure S1A). In parallel, we performed BONCAT experiments on S6K1 KO hippocampal slices, where the application of these inhibitors had no appreciable effect on global translation and phospho-S6 levels (Supplementary Figure S1B). Next, we used an adapted FUNCAT method (17) to measure newly synthesized proteins in hippocampal slices from WT and Fmr1 KO mice treated with PF-4708671 and FS-115. Both S6K1 inhibitors reduced protein synthesis in CA1 neurons in WT and Fmr1 KO mice (Figure $1 \mathrm{a}-\mathrm{c}$ ). To determine whether the effects of the S6K1 inhibitors on protein synthesis were specific to the hippocampus, we treated cortical lysates prepared from WT and Fmr1 KO littermates acutely with PF-4708671 and FS-115, and measured protein synthesis rates using BONCAT (Figure 1d and Supplementary Figure S1C). The inhibitors very rapidly reduced rates of AHA incorporation in the lysates, indicating that both inhibitors were effective at reducing exaggerated levels of translation in FXS model mice by specifically inhibiting S6K1.

\section{In vivo Administration of PF-4708671 and FS-115 Differentially Affect the Phosphorylation of Downstream Targets of S6K1}

We next tested the two inhibitors in their ability to suppress S6K1 activity in vivo. The two S6K1 inhibitors are structurally dissimilar. The $\mathrm{IC}_{50}$ values for S6K1 are substantially lower for FS-115 compared to PF-4708671, although PF-4708671 inhibits $\mathrm{S} 6 \mathrm{~K} 2$, a related kinase less critical for translational control, to lesser effect (Supplementary Table S1). FS-115 is orally bioavailable, whereas PF-4708671 can be delivered via i.p. injection to inhibit S6K1 in the brain (Huynh et al, 2014). Comparing the pharmacokinetic profiles of PF-4708671 and FS-115, we found that the two S6K1 inhibitors had differential brain-to-blood distribution profiles. One-hour post administration, PF-4708671 localized preferentially in the periphery, whereas FS-115 had a significantly higher brain accumulation (Supplementary Table S2). Although a dose response in vivo for PF-4708671 was described previously (Huynh et al, 2014), we retested doses ranging from 10 to $100 \mathrm{mg} / \mathrm{kg}$, and monitored the phosphorylation of several S6K1 substrates and downstream effectors, including S6, eIF4B, and eEF2, in whole hippocampal lysates from WT and Fmr1 KO mice for FS-115 (Supplementary Figure S1E). We found no detectable impact on phospho-eIF4B (Supplementary Figure S2E and F), suggesting that the influence of $\mathrm{S} 6 \mathrm{~K} 1$ inhibition on translation initiation was largely unaffected. Phosphorylation of S6 and eEF2 were most sensitive to changes in drug concentrations, and we limited our subsequent examination to these molecules. As in Huynh et al (2014), we found that an acute dose of PF-4708671 (50 mg/kg) resulted in a significant reduction in the phosphorylation of $\mathrm{S} 6$ in both genotypes (Supplementary Figure S2A and B) but had little effect on phosphorylated eEF2. A single gavage of FS-115 (100 mg/kg) resulted in similar effects on S6 and eEF2 phosphorylation (Supplementary Figure S2C and D). Interestingly, we found large variability in p-eEF2 levels in all genotypes upon acute treatments, especially oral gavages.

Although single dose effects of S6K1 inhibition are informative about rapid signaling modulation, viable therapeutic regimens targeting S6K1 would likely require chronic treatments. We therefore standardized optimal doses and durations of administration of both S6K1 inhibitors considering animal health, locomotor activity, and biochemical efficacy. A chronic 10-day treatment with PF-4708671 (25 mg/kg) significantly elevated eEF2 phosphorylation, but did not significantly reduce $\mathrm{S} 6$ phosphorylation in Fmr1 KO mice (Figure $2 \mathrm{a}$ and $\mathrm{b}$ ). In contrast, mice treated with FS-115 $(25 \mathrm{mg} / \mathrm{kg}$ ) for 5 days significantly reduced S6 phosphorylation in both WT and Fmr1 KO mice, whereas eEF2 phosphorylation was elevated specifically in Fmr1 KO mice (Figure $2 \mathrm{c}$ and $\mathrm{d}$ ). Notably, consistent with the enhanced eEF2 phosphorylation, we found that both S6K1 inhibitors significantly depressed protein synthesis in cortical lysates of Fmr1 KO mice without appreciably impacting WT mice (Figure 2e and f; Supplementary Figure S1D). Overall, these results indicate that chronic $\mathrm{S} 6 \mathrm{~K} 1$ inhibition impacts translation in the brains of Fmr1 KO mice differently than in WT mice, most likely by enhancing eEF2 phosphorylation and by extension, translation elongation. 
a

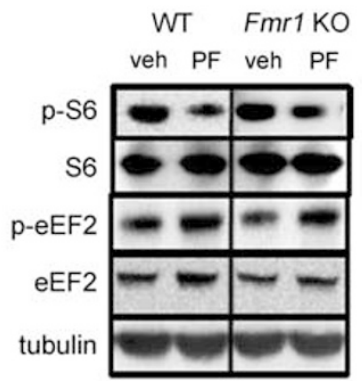

b

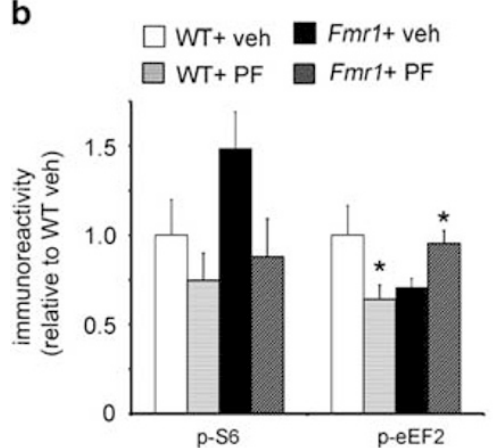

C
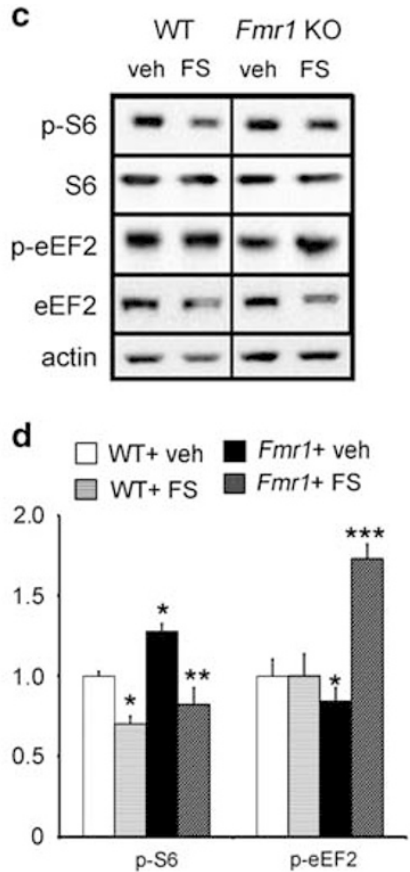

e

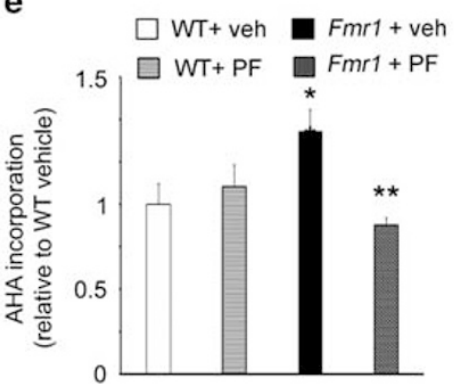

f

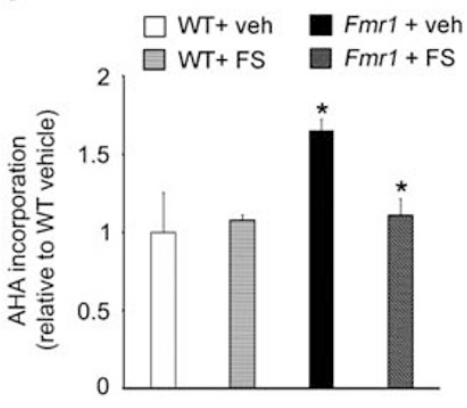

Figure 2 Chronic in vivo administration of PF-470867I and FS-I I 5 differentially affects the phosphorylation of translation control molecules. (a and b) Chronic treatment of WT and Fmrl KO mice with PF-470867 I significantly alters eEF2 phosphorylation, but not S6 phosphorylation, in a genotype-specific manner. $n=5$ mice per genotype per treatment; * $p<0.05$ with two-way ANOVA (genotype $x$ treatment), followed by Tukey's post hoc test. Significant interaction effect was found: $F(3,19)=5.87, F_{\text {crit }}=4.49$. (c and d) Five-day treatment of WT and Fmr I KO mice with FS-II5 significantly decreased S6 phosphorylation and increased eEF2 phosphorylation. $n=6-9$ mice per genotype per treatment; *p $<0.05$ and *** $p<0.00$ I with two-way ANOVA (genotype $x$ treatment), followed by Tukey's post hoc test. For both pS6 and eEF2, significant effect of interaction was found: $F(3.23)=5.008, F_{\text {crit }}=4.35$ for pS6; $F(3,32)=9.508, F_{c r i t}=4.14$. (e and f) BONCAT AHA incorporation in cortical lysates of mice treated with vehicle, PF-470867| or FS- I I5. $n=4$ mice per genotype per treatment. $*$ p $<0.05$ and $* *$ $p<0.0$ I by two-way ANOVA (genotype $\times$ treatment), followed by Bonferroni's post hoc test. Significant interaction effect was obtained $F(3,15)=5.81$ I, $F_{\text {crit }}=5.3$ I. Error bars represent SEM in (b), (d), and ( $f$. See also Supplementary Figure S2 for elF4B phosphorylation levels with acute treatment of PF-470867| and FS-II5.

\section{S6K1 Inhibitors Reverse ASD-Associated Deficits in Social Novelty Behavior and Behavioral Inflexibility in Fmr1 KO Mice}

ASD is currently diagnosed by the presentation of deficits in social development, communication, and repetitive behavior/ restricted interests (DSM V). We previously showed that the genetic ablation of S6K1 in Fmrl KO mice prevented impaired social novelty behavior and behavioral inflexibility while leaving stereotypic behaviors unaltered (Bhattacharya et al, 2012). Therefore, we determined whether adult Fmr1 $\mathrm{KO}$ mice treated with either S6K1 inhibitor could reverse the same behavioral abnormalities. We used a chronic 10-day dosing regimen of PF-4708671 after which behavioral tests were initiated. Impaired social novelty behavior was corrected in PF-470867-treated Fmrl KO mice (Figure 3a), with no effect on mouse $v s$ object preference (Supplementary Figure S3A and C). In contrast, treatment of the Fmr1 KO mice with PF-4708671 had no effect on increased marbleburying, a measure of repetitive behavior (Figure 3b). In addition, the PF-4708671-treated Fmr1 KO mice showed increased behavioral flexibility in the Y-maze choice reversal task (Figure 3c). We carried out the same battery of behavioral tests on mice given a shorter period of oral gavage treatment with FS-115 owing to its higher brain accumulation (Supplementary Table S2). We found that FS-115 treatment also reversed the impairment in social novelty behavior displayed by Fmr1 KO mice (Figure 3d) and rescued their behavioral inflexibility in the Y-maze (Figure 3f). To our surprise, we also observed a significant decrease in marble-burying activity of FS-115-treated Fmr1 KO mice (Figure 3e). Thus, chronic treatment with both S6K1 inhibitors was successful in reversing two common ASD-related behavioral phenotypes displayed by the FXS model mice, while differing in their effects on marbleburying behavior.

\section{Dendritic Spine Abnormalities in Fmr1 KO Mice are Reversed by Chronic Blockade of S6K1}

We proceeded to determine whether PF-4708671 and FS-115 could reverse the dendritic spine abnormalities displayed by the FXS model mice. Previously, we found that the genetic deletion of S6K1 corrected both increased dendritic spine density and increased immature filopodia-like spines in FXS mice (Bhattacharya et al, 2012). In the experiments with PF-4708671, our dendritic spine analyses revealed no appreciable change in total spine number per unit of dendritic length in Fmr1 KO mice, but yielded a significant reduction in filopodia-like spines and a concomitant increase in mature stubby/mushroom spines (Figure $4 \mathrm{a}$ and $\mathrm{b}$ ). We obtained similar results with $F m r 1$ KO mice treated chronically with FS-115 (Figure 4c and d). Intriguingly, we also noticed a trend of increased filopodial spines in neurons 
from WT mice treated with FS-115 (Figure 4c and d), suggesting that there are S6K1 inhibitor-specific effects on dendritic morphology in WT mice. Nevertheless, these results indicate that the increase in filopodia-like spines in Fmr1 KO mice are reversed by inhibiting S6K1 with either PF-4708671 or FS-115.
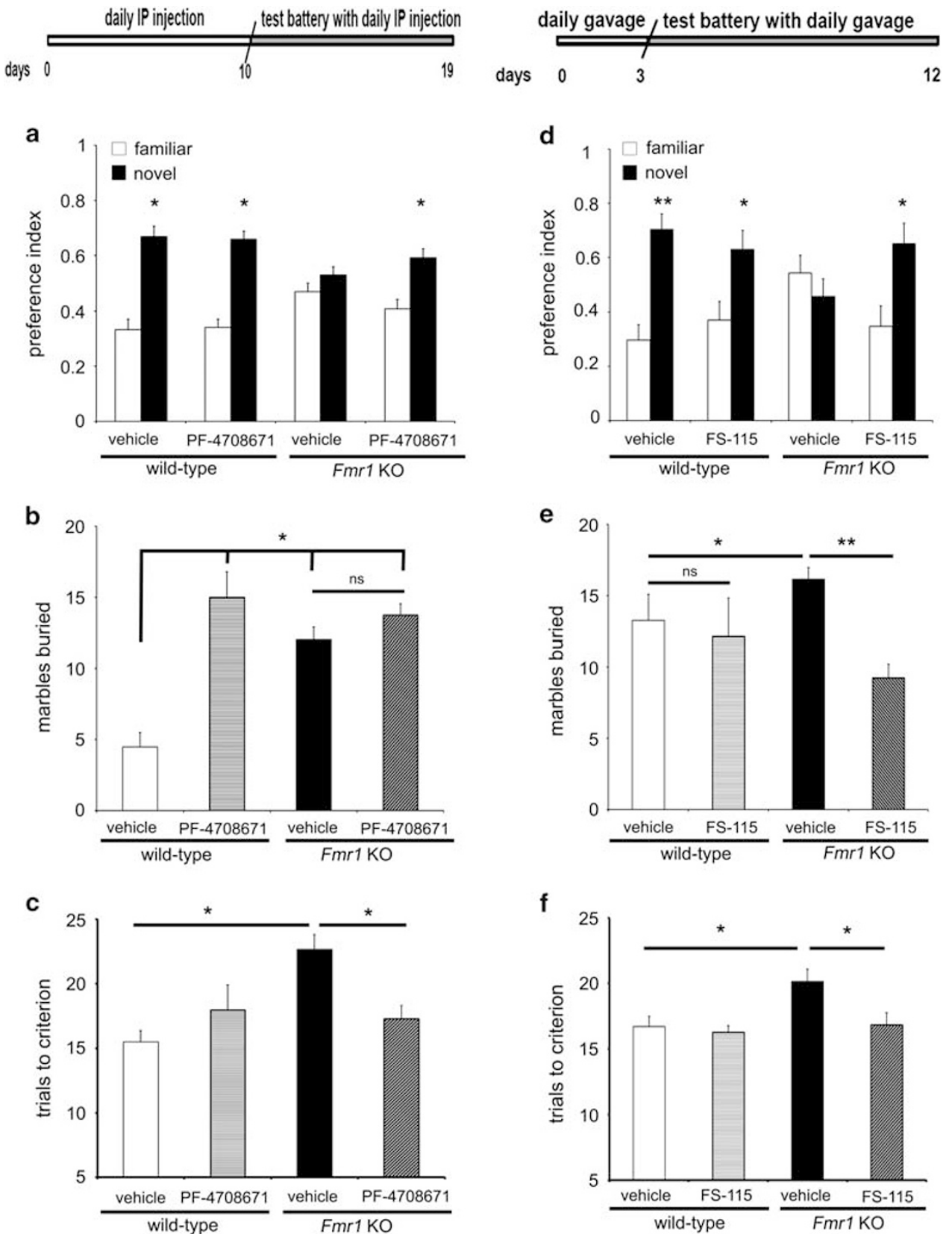

Figure 3 Chronic treatment of Fmrl KO mice with PF-470867I and FS- II 5 reversed impaired social behavior and behavioral inflexibility with differential effects on marble-burying behavior. (Top) Schematic showing timeline and treatment scheme for both PF-470867I- and FS-II5-treated cohorts of mice. PF-470867 I was injected daily for 10 days, followed by experiments conducted over the next 9 days. FS- I I5-treated cohorts were gavaged for 3 days, followed by experiments conducted over the next 9 days. Please see Behavioral Methods for details. (a and d) Interaction preference of wild-type and Fmr I

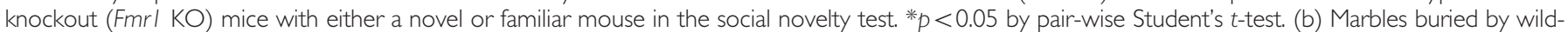
type and Fmrl KO mice treated with either vehicle or PF-470867I. $* p<0.05$ and $* * p<0.01$ compared to wild-type+vehicle by two-way ANOVA (genotype $x$ treatment), followed by Bonferroni-Dunn post hoc analyses. Strong main effects were found across treatments and across genotypes separately: $\mathrm{F}(1,32)=7.57, \mathrm{~F}_{\text {crit }}=4.15$. (c) Trials to criterion required by PF-470867I treatment group in the $\mathrm{Y}$-maze choice arm reversal test. * $p<0.05$ by two-way ANOVA (genotype $x$ treatment), followed by Bonferroni-Dunn post hoc analyses. Significant interaction was found: $F(3,32)=18.6, F_{c r i t}=4.15$. (e) Marbleburying behavior of wild-type and Fmrl KO mice treated with FS-I I5. $* p<0.05$ for wild-type+vehicle vs Fmrl $\mathrm{KO}+$ vehicle and $* * p<0.01$ for Fmrl $\mathrm{KO}$ +vehicle vs Fmr I KO+FS- II 5 by two-way ANOVA (genotype $x$ treatment), followed by Bonferroni-Dunn post hoc analyses. Significant main effects were found across samples separately: $F(1,28)=5.24, F_{\text {crit }}=4$. 19. (f) Trials to criterion required by each FS-I I 5 treatment group in the $Y$-maze choice arm reversal test. $* 0$ p 0.05 for comparison between wild-type+vehicle vs Fmrl KO+vehicle and Fmrl KO+vehicle vs Fmrl KO+FS-II5 by two-way ANOVA (genotype $x$ treatment), followed by Bonferroni-Dunn post hoc analyses. Significant main effects were seen across genotypes: $F(1,24)=6.42, F_{\text {crit }}=4.25$. For all tests $n=10-12$ mice per treatment per genotype. Error bars denote SEM. See also Supplementary Figure S3. 

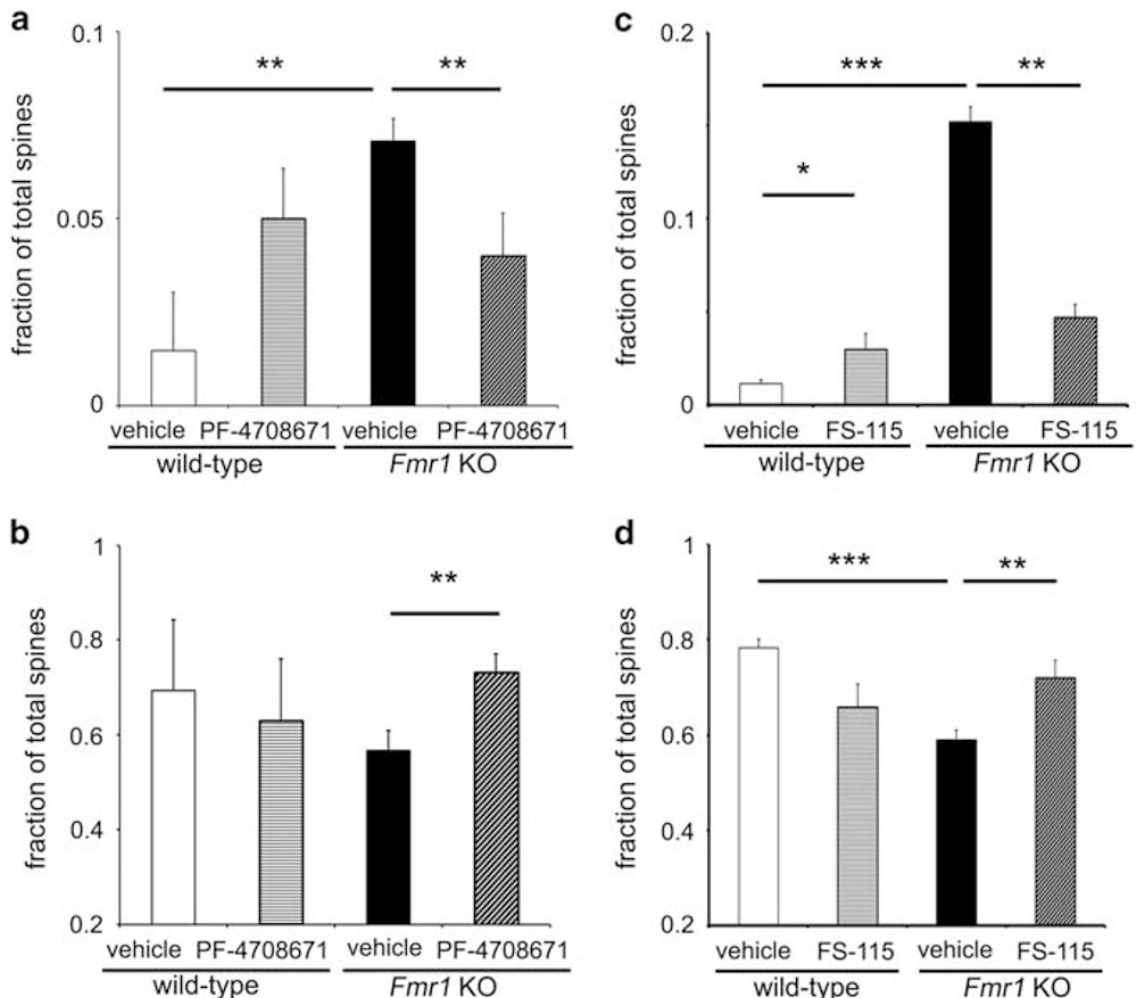

Figure 4 Inhibition of S6KI by PF-470867I and FS-I I 5 in FmrI KO mice rescues abnormal spine morphology. (a) Abnormal filopodial morphology in Fmr I knockout (Fmrl KO) mice was normalized by chronic administration of PF-470867l. *** $<0.0$ I by Friedman non-parametric ANOVA, followed by Dunn's multiple comparison test. (b) Decreased mature spine morphology (stubby and mushroom spines) of Fmrl KO mice was normalized by chronic administration of PF-470867I. *** $<<0.01$ by Kruskal-Wallis non-parametric ANOVA, followed by Dunn's multiple comparison test. (c) Filopodial morphology of Fmrl KO

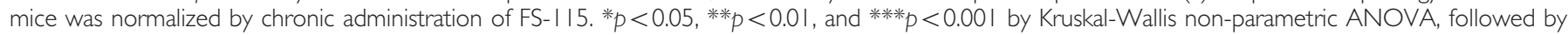
Dunn's multiple comparison test. (d) Decreased mature spine morphology (stubby and mushroom spines) in Fmrl KO mice was normalized by chronic administration of FS-1 I 5. *** $<0.01$ and **** $p<0.00$ I by Kruskal-Wallis non-parametric ANOVA followed by Dunn's multiple comparison test. $n=3$ mice (3-5 neurons per mouse, $n=9-15$ neurons per condition) for cohorts treated with either vehicle or PF-470867I. $n=3$ mice (4-6 neurons per mouse, $n=12-18$ neurons per condition) for cohorts treated with either vehicle or FS- I I . For all graphs, error bars denote SEM. See also Supplementary Figure S4.

\section{Differential Accumulation of S6K1 Inhibitors Influences Peripheral Effects of Weight Gain and Macroorchidsm of FXS Model Mice}

Because S6K1 is ubiquitously expressed and given the systemic delivery routes we used for both S6K1 inhibitors, we also examined peripheral phenotypes displayed by the FXS model mice. S6K1 KO mice are visibly smaller than their WT littermates (Shima et al, 1998), which is opposite of the increased weight gain and macroorchidism displayed by Fmr1 KO mice (Santoro et al, 2012; Hagerman et al, 1983). Furthermore, these peripheral phenotypes exhibited by the FXS model mice were prevented by the genetic reduction of S6K1 (Bhattacharya et al, 2012). We found no effect on body weight in PF-4708671-treated Fmr1 KO mice (Figure 5a). However, there was a significant reduction in testicular weight in PF-4708671-treated Fmr1 KO mice (Figure 5b). In contrast, treatment with FS-115 significantly decreased the body weight of the Fmr1 KO mice (Figure 5c). In addition, the enlarged testicular phenotype in FXS model mice was reduced by treatment with FS-115 (Figure 5d). Taken together, our data suggest that peripheral $v s$ brain localization of S6K1 inhibitor predicts effects on body weight but not testicular size in FXS model mice.

\section{DISCUSSION}

Altered mTORC1 signaling plays a role in diverse brain disorders ranging from schizophrenia, Alzheimer's disease, epilepsy, and neurodevelopmental disorders such as FXS and tuberous sclerosis (Lipton and Sahin, 2014). Although rapamycin and everolimus are FDA-approved to target mTORC1 signaling, the fact that mTORC1 plays a critical role in other cellular processes apart from translation, could limit their use to extreme conditions such as transplant rejection and anticancer treatments (Lipton and Sahin 2014; Hoeffer and Klann, 2010). In this scenario, it is worthwhile to explore treatment strategies and chemical compounds that specifically target the mTORC1 effectors $4 \mathrm{E}-\mathrm{BP}$, which represses eIF4E, and S6K1 for conditions that have a clear molecular basis for dysregulated translation. Recently, there has been a marked increase in the discovery of specific S6K1 inhibitors (Niwa et al, 2014; Couty et al, 2013), primarily fueled by the direct implication of S6K1 in various types of cancers and indirectly in obesity (Holz, 2012; Selman et al, 2009). Although it is encouraging to note these developments, the need to characterize the behavior of these new chemicals in the brain and related side effects becomes significant. In this study, we evaluated the effects of inhibiting S6K1 in the brain and whether this can ameliorate 

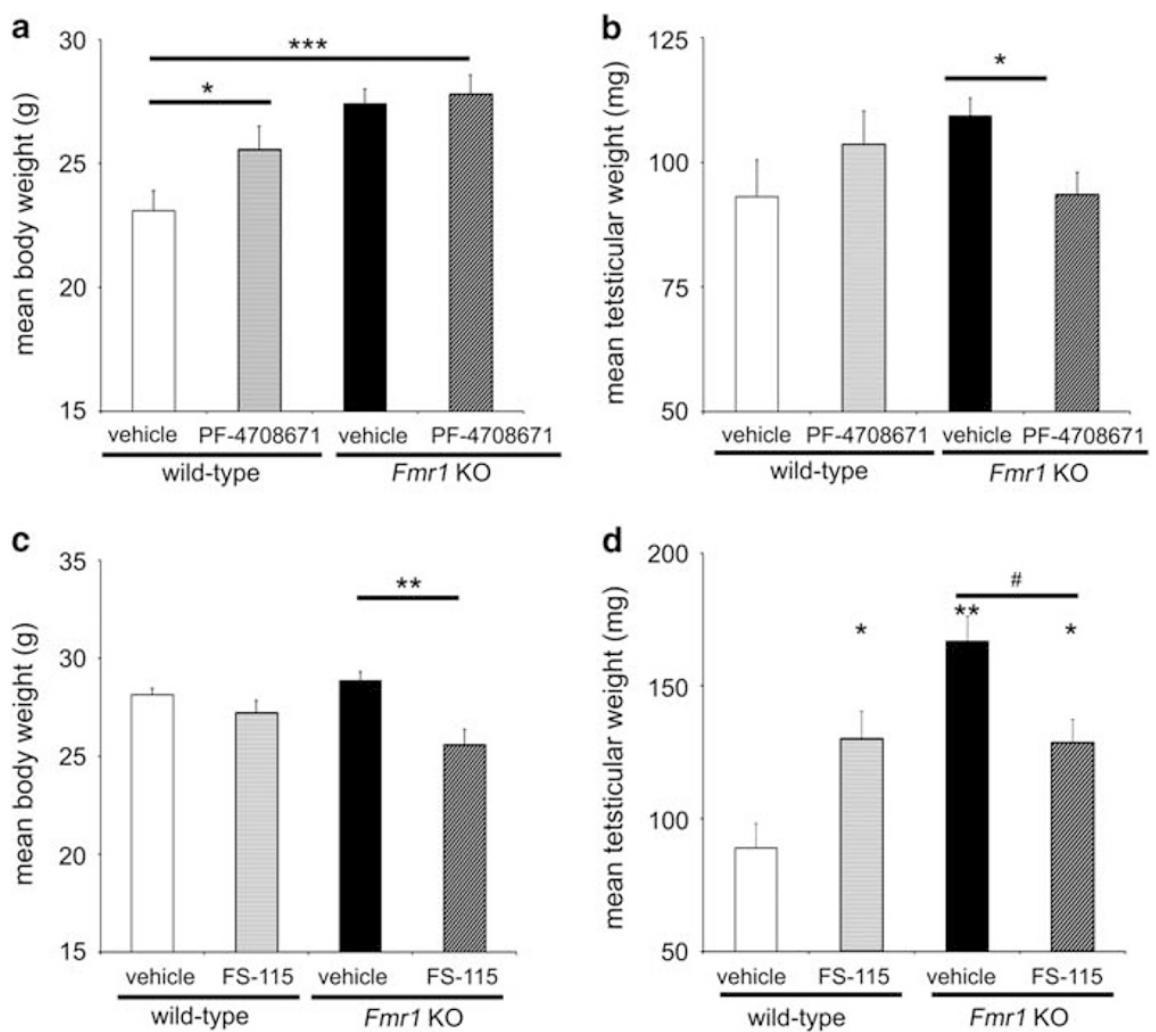

Figure 5 Chronic treatment with PF-470867I and FS- I 5 have differential effects on peripheral phenotypes displayed by Fmr I KO mice. (a) Average body weights of wild-type and Fmrl knockout (Fmrl KO) mice treated with either vehicle or PF-470867I. $n=|3-| 4$ mice per genotype per group. * $<<0.05$ and **** $p<0.00$ I by two-way ANOVA (genotype $x$ treatment), followed by Bonferroni post hoc analyses. There was no significant effect of interaction, but significant main effects across treatment and genotypes: $F(1,55)=4.48$ for wild-type+vehicle vs wild-type+PF-470867l; $F(1,55)=23.48$ for wild-type+vehicle vs Fmrl KO+vehicle and Fmrl KO+PF-470867I, $F_{\text {crit }}=4.02$. (b) Average testicular weights of wild-type and Fmrl KO mice treated with either vehicle or PF-470867I. $n=9$ mice per genotype per group. $* 0<0.05$ by two-way ANOVA (genotype $x$ treatment), followed by Bonferroni-Dunn post hoc comparison. There was a significant effect of interaction, $\mathrm{F}_{3,20}=20.5, \mathrm{~F}_{\text {crit }}=4.35$ for Fmrl KO+vehicle vs Fmrl KO+PF-470867I. (c) Average body weights of wild-type and Fmrl KO mice treated with either vehicle or FS-II5. $n=12$ mice per genotype per group. *** $<0.01$ by two-way ANOVA (genotype $\times$ treatment), followed by Bonferroni-Dunn post hoc comparison. There was a significant effect of interaction: $F(3,44)=16.1$, $F_{\text {crit }}=4.06$. (d) Average testicular weights of wild-type and Fmrl KO mice treated with either vehicle or FS- I I 5. $n=6$ mice per genotype per group. * $p<0.05$, ** $p<0.0$ I when compared to wild-type +vehicle, and ${ }^{\#} p<0.05$ by two-way ANOVA (genotype $x$ treatment), followed by Bonferroni-Dunn post hoc comparison. There was significant effect of interaction, $F(3,20)=7.3, F_{\text {crit }}=4.35$.

disease-related phenotypes in FXS model mice. We tested two chemically dissimilar S6K1 inhibitors with differing activity profiles and found that both could not only reverse a common set of biochemical, behavioral, neuronal morphology, and peripheral phenotypes, but also had divergent effects on weight gain and marble-burying behavior displayed by FXS model mice. To our knowledge, this is the first study to test the therapeutic potential of S6K1 inhibitors in a mouse model for a syndromic neurological disorder.

Dysregulated translation is believed to be one of the fundamental causes of FXS pathophysiology (Santoro et al, 2012; Darnell and Klann, 2013; Richter et al, 2015). Multiple recent studies have shown that pharmacological treatments at several signaling and regulation points reset translational homeostasis to prevent/reverse synaptic and behavioral phenotypes displayed by FXS mice (Bhattacharya et al, 2012; Gkogkas et al, 2014; Gross et al, 2015a, b; Michalon et al, 2012; Osterweil et al, 2013; Sidhu et al, 2014; Udagawa et al, 2013). Among these, only a few studies targeted translational control genetically and/or pharmacologically (Bhattacharya et al, 2012; Gkogkas et al, 2014; Udagawa et al,
2013). Because systemically targeting the protein synthesis regulatory machinery could result in adverse effects, it is important to identify and target those molecules that lie at signaling nodes that funnel multiple upstream signaling pathways to translation factors and ribosomal proteins, and have minimal pleiotropic effects on other cellular processes. Previously, we genetically ablated S6K1, which fulfills most of these constraints, and found that it could prevent multiple phenotypes in FXS mice (Bhattacharya et al, 2012). This present study is a head-to-head comparison with our previous genetic studies to evaluate which phenotypes could be normalized by small molecule inhibitors of S6K1. This also directed our choice of brain areas and assays in which we had observed benefits of genetically reducing S6K1 in FXS model mice (Bhattacharya et al, 2012). A major difference between the previous study and the findings herein is that genetically reducing S6K1 in FXS model mice prevents many maladaptive changes that begin with embryogenesis, whereas the pharmacological inhibition of S6K1 reverses the consequences of S6K1 activation on more immediate time scales. The latter not only provides insight into the dynamic nature of translational control by S6K1, but also tests the ability of 
S6K1 inhibitors to reset aberrant phenotypes in FXS mice that form throughout development. This type of approach is more valuable therapeutically given that individuals with FXS likely would receive treatment after the disease was fully manifested. Notably, our results demonstrate reversal of phenotypes in adult FXS mice, which could be relevant for the recruitment of older children and young adults in future clinical trials of FXS. Thus, regulators of translation control may offer a novel avenue to treat individuals with FXS.

Our biochemical results (Figure 2; Supplementary Figure S2; Supplementary Tables S1 and S2) not only indicate clear functional selectivity of inhibiting S6K1 over different time scales, but also demonstrate differences in drug actions of PF-4708671 and FS-115. For example, phospho-S6 (240/44), was consistently downregulated by acute treatment with either inhibitor, but was only significantly decreased by chronic FS-115 treatment (Figure 2c and d). Although S6 is important for translation, the precise function of its phosphorylation remains unresolved. Therefore, modulation of S6 phosphorylation does not predict whether S6K1 inhibition preferentially impacts either initiation or elongation. In contrast, chronic dosing of PF-4708671 and FS-115 both modulated eEF2 phosphorylation in Fmr1 KO mice (Figure $2 \mathrm{c}$ and $\mathrm{d}$ ), suggesting that both inhibitors have to accumulate in mice in order to decrease translation elongation. We also found a more profound decrease in basal rates of translation in cortical lysates of Fmrl KO mice that were chronically treated with the S6K1 inhibitors (Figure 2e and f). The lysates from chronically treated WT mice showed high variability in not only phosphorylation of S6 and eEF2 but also protein synthesis rates, and may allude to greater signaling redundancy in WT mice as compared to FXS model mice. This merits further investigation. In summary, our biochemical experiments provide insight into the mechanism by which pharmacological inhibition of S6K1 puts a brake on exaggerated translation in Fmr1 KO mice.

We examined the impact of both S6K1 inhibitors on behavioral phenotypes displayed by FXS model mice that were corrected by the genetic reduction of S6K1. Despite the different pharmacological profiles of PF-4708671 and FS-115, both S6K1 inhibitors were able to reverse impairments in social novelty and behavioral inflexibility in the Y-maze choice arm reversal test displayed by Fmr1 KO mice (Figure 3a, c, d, and f). In addition, marble-burying was not impacted in Fmrl KO mice treated with PF-4708671 (Figure 3b), which is consistent with our previous results with genetic reduction of S6K1 (Bhattacharya et al, 2012). Hence, the two inhibitors and the genetic approach rescue a core set of deficits arguing for a clear involvement of hyperactive S6K1 in the etiology of these behaviors in FXS model mice, and may inform selection of outcome measure for any future human trials involving S6K1 inhibitors. It was also equally likely that either inhibitor would have off-target unforeseen effects as shown for FS-115-treated mice in marble-burying behavior test (Figure 3e), which could be due to the effects of continued brain accumulation of FS-115 that impacts additional brain circuits in the FXS mice that are responsible for this behavior.

We reported corrected abnormal spine density and morphology in Fmr1 KO mice with a genetic reduction of S6K1 (Bhattacharya et al, 2012). In PF-4708671-treated FXS model mice, we found a similar decrease in the fraction of filopodial spines, demonstrating that even with modest brain accumulation, PF-4708671 was able to reverse the immature spine phenotype. With the more brain-localized FS-115, we obtained a robust decrease in the fraction of filopodial spines in drug-treated Fmr1 KO mice and a concomitant increase in the fraction of mature spines. Taken together, these findings suggest that modulating S6K1 activity impacts the spine morphology of CA1 hippocampal neurons in Fmr1 KO mice. Interestingly, spine density was not altered in either S6K1 inhibitor-treated Fmr1 KO mice possibly due to background differences (Pop et al, 2013) or because spine density may be developmentally constrained that is refractory to manipulation in adult mice.

The impact of the S6K1 inhibitors on the peripheral phenotypes displayed by FXS model mice suggest that body weight and testicular size are differentially regulated by S6K1. The more peripherally available PF-4708671 was ineffective in decreasing the increased body weight of Fmr1 KO mice, but significantly reduced macroorchidism in the same mice. These results suggest that the regulation of body weight requires neural S6K1 signaling, whereas testicular size requires peripheral S6K1 signaling. Consistent with this notion, there was a significant decline in the body weights of WT and Fmr1 KO mice treated with FS-115 (Figures 5c and d), which also was successful in reversing macroorchidism in FXS mice (Figure 5d).

In summary, using PF-4708671 and FS-115, we provide an initial characterization of the effects of S6K1 inhibition in the brain, and establish their therapeutic potential by correcting multiple disease-associated phenotypes in FXS model mice. Our findings support the idea that resetting translation homeostasis by targeting translational control molecules in FXS is beneficial and should be the focus of further drug development.

\section{FUNDING AND DISCLOSURE}

$\mathrm{EK}, \mathrm{AB}, \mathrm{MM}, \mathrm{AC}$, and $\mathrm{CM}$ declare no conflict of interest. RGB and DWW are employees of, and have a financial interest in Sentinel Oncology. DGS was a full-time employee of Pfizer when the project was initiated. This work was supported by NIH grants NS034007, NS047384, and HD082013 (to EK), the FRAXA Research Foundation and the Charles H. Revson Foundation (to AB), and NIH-BPEndure Grant R25NS080686 (to AC).

\section{ACKNOWLEDGMENTS}

The authors would like to acknowledge the technical assistance provided by Ms Nicole Zeak at Center for Neural Science at New York University and Ms Kari Fonseca at Pfizer Neuroscience.

\section{REFERENCES}

American Psychiatric Association (2013). Diagnostic and Statistical Manual of Mental Disorders, 5th edn. Americal Psychiatric Publishing: Arlington, VA.

Bhattacharya A, Kaphzan H, Alvarez-Dieppa AC, Murphy JP, Pierre P, Klann E (2012). Genetic removal of p70 S6 kinase 1 corrects molecular, synaptic, and behavioral phenotypes in fragile X syndrome mice. Neuron 76: 325-337. 
Bowling H, Bhattacharya A, Zhang G, Lebowitz JZ, Alam D, Smith $\mathrm{P}$ et al (2016). BONLAC: a combinatorial proteomic technique to measure stimulus-induced translational profiles in brain slices. Neuropharmacology 100: 76-89.

Costa-Mattioli M, Sossin WS, Klann E, Sonenberg N (2009). Translational control of long-lasting synaptic plasticity and memory. Neuron 61: 10-26.

Couty S, Westwood IM, Kalusa A, Cano C, Travers J, Boxall K et al (2013). The discovery of potent ribosomal S6 kinase inhibitors by high-throughput screening and structure-guided drug design. Oncotarget 4: 1647-1661.

Darnell JC, Klann E (2013). The translation of translational control by FMRP: therapeutic targets for FXS. Nat Neurosci 16: 1530-1536.

Eyre TA, Collins GP, Goldstone AH, Cwynarski K (2014). Time now to TORC the TORC? New developments in mTOR pathway inhibition in lymphoid malignancies. $\mathrm{Br} J$ Haematol 166: 336-351.

Gkogkas CG, Khoutorsky A, Cao R, Jafarnejad SM, Prager-Khoutorsky M, Giannakas N et al (2014). Pharmacogenetic inhibition of eIF4E-dependent Mmp9 mRNA translation reverses fragile $\mathrm{X}$ syndrome-like phenotypes. Cell Rep 9: $1742-1755$.

Gross C, Chang CW, Kelly SM, Bhattacharya A, McBride SM, Danielson SW et al (2015a). Increased expression of the PI3K enhancer PIKE mediates deficits in synaptic plasticity and behavior in fragile X syndreome. Cell Rep 11: 727-736.

Gross C, Nakamoto M, Yao X, Chan CB, Yim SY, Ye K et al (2010). Excess phosphoinositide 3-kinase subunit synthesis and activity as a novel therapeutic target in fragile X syndrome. J Neurosci $\mathbf{3 0}$ : 10624-10638.

Gross C, Raj N, Molinaro G, Allen AG, Whyte AJ, Gibson JR et al (2015b). Selective role of the catalytic PI3K subunit p110 $\beta$ in impaired higher order cognition in fragile X syndrome. Cell Rep 11: 681-688.

Hagerman RJ, McBogg P, Hagerman PJ (1983). The fragile X syndrome: history, diagnosis, and treatment. J Dev Behav Pediatr 4: $122-130$.

Hoeffer CA, Klann E (2010). mTOR signaling: at the crossroads of plasticity, memory and disease. Trends Neurosci 33: 67-75.

Hoeffer CA, Sanchez E, Hagerman RJ, Mu Y, Nguyen DV, Wong H et al (2012). Altered mTOR signaling and enhanced CYFIP2 expression levels in subjects with fragile X syndrome. Genes Brain Behav 11: 332-341.

Holz MK (2012). The role of S6K1 in ER-positive breast cancer. Cell Cycle 11: 3159-3165.

Huynh TN, Santini E, Klann E (2014). Requirement of mammalian target of rapamycin complex 1 downstream effectors in cued fear memory reconsolidation and its persistence. J Neurosci 34: 9034-9039.

Kumari D, Bhattacharya A, Nadel J, Moulton K, Zeak NM, Glicksman A et al (2014). Identification of fragile X syndromespecific molecular markers in human fibroblasts: a useful model to test the efficacy of therapeutic drugs. Hum Mutat 35: 1485-1494.
Lipton JO, Sahin M (2014). The neurology of mTOR. Neuron 84: 275-291.

Magnuson B, Ekim B, Fingar DC (2012). Regulation and function of ribosomal protein S6 kinase (S6K) within mTOR signalling networks. Biochem J 441: 1-21.

Michalon A, Sidorov M, Ballard TM, Ozmen L, Spooren W, Wettstein JG et al (2012). Chronic pharmacological mGlu5 inhibition corrects fragile X in adult mice. Neuron 74: 49-56.

Niwa H, Mikuni J, Sasaki S, Tomabechi Y, Honda K, Ikeda M et al (2014). Crystal structures of the S6K1 kinase domain in complexes with inhibitors. J Struct Funct Genomics 15: 153-164.

Osterweil EK, Chuang SC, Chubykin AA, Sidorov M, Bianchi R, Wong RK et al (2013). Lovastatin corrects excess protein synthesis and prevents epileptogenesis in a mouse model of fragile X syndrome. Neuron 77: 243-250.

Osterweil EK, Krueger DD, Reinhold K, Bear MF (2010). Hypersensitivity to mGluR5 and ERK1/2 leads to excessive protein synthesis in the hippocampus of a mouse model of fragile X syndrome. J Neurosci 30: 15616-15627.

Pearce LR, Alton GR, Richter DT, Kath JC, Lingardo L, Chapman J et al (2010). Characterization of PF-4708671, a novel and highly specific inhibitor of p70 ribosomal S6 kinase (S6K1). Biochem J 431: 245-255.

Pop AS, Levenga J, de Esch CE, Buijsen RA, Nieuwenhuizen IM, Li T (2013). Rescue of dendritic spine phenotype in Fmr1 KO mice with the mGluR5 antagonist AFQ056/mavoglurant. Psychopharmacology (Berl) 231: 1227-1235.

Richter JD, Bassell GJ, Klann E (2015). Dysregulation and restoration of translational homeostasis in fragile $\mathrm{X}$ syndrome. Nat Rev Neurosci 16: 595-605.

Santoro MR, Bray SM, Warren ST (2012). Molecular mechanisms of fragile X syndrome: a twenty-year perspective. Annu Rev Pathol 7: 219-245.

Selman C, Tullet JM, Wieser D, Irvine E, Lingard SJ, Choudhury AI et al (2009). Ribosomal protein S6 kinase 1 signaling regulates mammalian life span. Science 326: 140-144.

Sharma A, Hoeffer CA, Takayasu Y, Miyawaki T, McBride SM, Klann E et al (2010). Dysregulation of mTOR signaling in fragile X syndrome. J Neurosci 30: 694-702.

Shima H, Pende M, Chen Y, Fumagalli S, Thomas G, Kozma SC (1998). Disruption of the p70(s6k)/p85(s6k) gene reveals a small mouse phenotype and a new functional S6 kinase. EMBO J 17: 6649-6659.

Sidhu H, Dansie LE, Hickmott PW, Ethell DW, Ethell IM (2014). Genetic removal of matrix metalloproteinase 9 rescues the symptoms of fragile X syndrome in a mouse model. J Neurosci $\mathbf{3 4}$ : 9867-9879.

Tolcher A, Goldman J, Patnaik A, Papadopoulos KP, Westwood P, Kelly CS et al (2014). A phase I trial of LY2584702 tosylate, a p70 S6 kinase inhibitor, in patients with advanced solid tumours. Eur J Cancer 50: 867-875.

Udagawa T, Farny NG, Jakovcevski M, Kaphzan H, Alarcon JM, Anilkumar S et al (2013). Genetic and acute CPEB1 depletion ameliorate fragile X pathophysiology. Nat Med 19: 1473-1477.

Supplementary Information accompanies the paper on the Neuropsychopharmacology website (http://www.nature.com/npp) 